\title{
CP violation summary
}

\section{Giancarlo D'Ambrosio}

INFN Sezione di Napoli

E-mail: gdambrosena.infn.it

\section{Daniela Rebuzzi}

University of Pavia

E-mail: daniela.rebuzzidcern.ch

\section{Hitoshi Yamamoto*}

Tohoku University

E-mail: yhtioshi@epx.phys.tohoku.ac.jp

In the two sessions devoted to $\mathrm{CP}$ violation, rich physics results have been reported by speakers from D0, CDF, LHCb, Belle, and BaBar. In addition, a useful phenomenological review has been given. There are a few measurements that show possible deviations from the standard model as well as those with significantly improved precisions. We briefly mention some highlights in this short summary.

The Xth Nicola Cabibbo International Conference on Heavy Quarks and Leptons, October 11-15, 2010

Frascati (Rome) Italy

\footnotetext{
* Speaker.
} 


\section{Mixing and semileptonic asymmetries}

The D0 experiment has reported a result on the dilepton asymmetry defined by

$$
A_{s l}^{b} \equiv \frac{N_{b}^{++}-N_{b}^{--}}{N_{b}^{++}+N_{b}^{--}}
$$

which is related to the wrong-sign single lepton asymmetries of $B_{d}$ and $B_{s}, a_{s l}^{d, s}$, by

$$
A_{s l}^{b}=(0.506 \pm 0.043) a_{s l}^{d}+(0.494 \pm 0.043) a_{s l}^{d} .
$$

The contributions from $B_{d}$ and $B_{s}$ are roughly equal since $B_{s}$ is produced less but its mixing is larger. After correcting for the asymmetries due to backgrounds and detector, it is measured to be

$$
A_{s l}^{b}=0.957 \pm 0.251_{\text {stat }} \pm 0.146_{\text {sys }} \% .
$$

Fig 1(left) shows the result in the 2-dimensional space of $a_{s l}^{s}$ vs $a_{s l}^{d}$. The deviation from SM is $3.2 \sigma$, while it is consistent with previous measurements. It is considered impossible that this deviation is reproduced by assumption of no tree-level new physics. At $\mathrm{LHCb}$, there are large production asymmetries due to $p p$ collision, the difference between $a_{s l}^{s}$ and $a_{s l}^{d}$, however, can be measured with small systematics. The expected resolution with $1 \mathrm{fb}^{-1}$ is shown in the figure as a yellow line.

Deviation from SM of the $B_{s}$ mixing can be parametrized by

$$
M_{12}^{\text {full }}=C_{B s} e^{2 i \phi_{B s}} M_{12}^{S M} .
$$

Current result for $B_{s}$ is shown in Fig 1 (right). While the absolute value of $M_{12}$ is consistent with $\mathrm{SM}$, its phase is $3.2 \sigma$ away from SM.
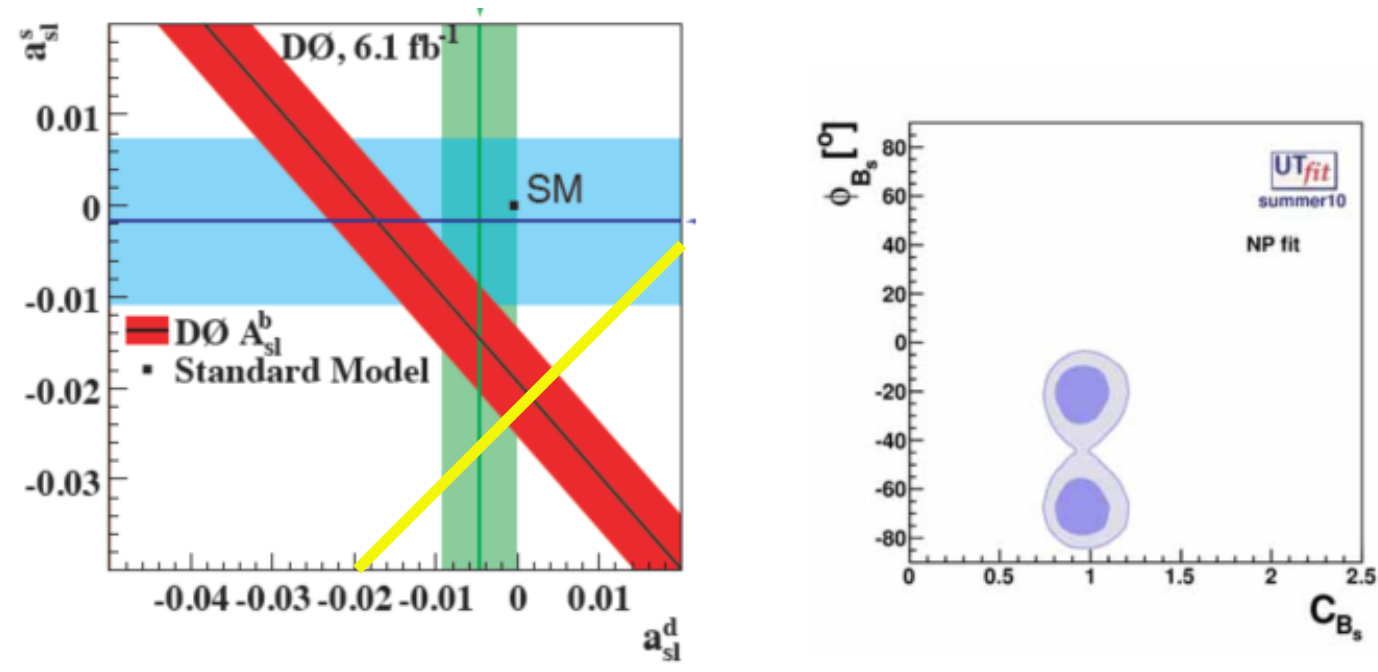

Figure 1: Left: Dilepton asymmetry measurement by D0. The blue band is a $a_{s l}^{s}$ measurement by D0 and the green band is the measurement of $a_{s l}^{d}$ by the B factories. Right: Deviations from the standard model of Bs mixing parameters. 


\section{Hadronic final states - time-dependent}

Using the final state $J / \Psi \phi, \mathrm{D} 0$ and $\mathrm{CDF}$ have measured the mixing phase $\phi_{s}\left(\sim-2 \beta_{s}\right)$ of $B_{s}$. The decay angular distribution allows separation of CP even and CP odd components. By fitting the decay time as well as the angular distribution, D0 has obtained $\phi_{s}=-0.76_{-0.36}^{+0.38} \pm 0.02$, and the SM expectation $(\sim-0.04)$ is well within its $95 \%$ confidence interval. The value obtained by $\mathrm{CDF}, \beta_{s}=(0.28,0.52) \cup(1.08,1.55) 68 \%$ confidence interval, is also consistent with SM within $1 \sigma$. These are based on $5 \sim 6 \mathrm{fb}^{-1}$ of luminosity and the statistical errors are expected to improve. The sensitivity on $\phi_{s}$ by LHCb will approach the SM value with $\sim 1 \mathrm{fb}^{-1}$ of luminosity.

As for $b \rightarrow s g$ time-dependent analyses, the naive world average of $\beta / \phi_{1}^{\text {eff }}$ for many $b \rightarrow s g$ modes is now consistent with the corresponding value of $b \rightarrow c \bar{c} s$ modes:

$$
\beta / \phi_{1}^{\text {eff }}=0.64 \pm 0.04(b \rightarrow s g), \quad 0.67 \pm 0.02(b \rightarrow c \bar{c} s) .
$$

The possible deviation from SM that was reported earlier seems to have disappeared.

\section{Hadronic final states - time-independent}

Direct CP asymmetry $A_{f}$ of a decay $B \rightarrow f$ is defined by

$$
A_{f} \equiv \frac{\Gamma(\bar{B} \rightarrow \bar{f})-\Gamma(B \rightarrow f)}{\Gamma(\bar{B} \rightarrow \bar{f})-\Gamma(B \rightarrow f)} .
$$

The so-called $K \pi$ puzzle is the discrepancy between $A_{f}$ 's of $B^{+} \rightarrow K^{+} \pi^{0}$ and $B^{0} \rightarrow K^{+} \pi^{-}$. Naive isospin argument indicates that the two asymmetries are the same. The measurements by Belle are

$$
\left\{\begin{array}{c}
A_{K^{+} \pi^{-}}=-0.094 \pm 0.018 \pm 0.008 \\
A_{K^{+} \pi^{0}}=0.07 \pm 0.03 \pm 0.01
\end{array}, \quad A_{K^{+} \pi^{0}}-A_{K^{+} \pi^{-}}=0.164 \pm 0.037\right.
$$

This discrepancy could be due to a new physics such as a new $b s Z$ interaction. However, it is also consistent with SM within hadronic uncertainty.

Measurements on the last of the three CP violating angles, $\phi_{3} / \gamma$, are getting to be more precise. Up to now, the best measurements come from analyses of $B^{+} \rightarrow D K^{+}$(and its CP conjugate mode) followed by $D \rightarrow K_{s} \pi^{+} \pi^{-}$where the interference in the Dalitz distribution make it possible to extract $\phi_{3} / \gamma$ and the strong phase separately. The latest results are

$$
\begin{cases}\gamma=\left(68_{-14}^{+15} \pm 4 \pm 3\right)^{\circ} & (\text { BaBar }) \\ \phi_{1}=\left(78_{-12}^{+11} \pm 4 \pm 9\right)^{\circ} & (\text { Belle })\end{cases}
$$

where the last errors are the systematic errors due to modeling of the 3-body $D^{0}$ decay. One way to avoid such model dependence is to use more than two 2-body final states of $D$ decay in $B^{+} \rightarrow D K^{+}$. This technique is called the Atwood-Dunietz-Soni (ADS) method, and the key mode is the highly suppressed mode $B^{+} \rightarrow D K^{+}$followed by $D \rightarrow K^{-} \pi^{+}$. The first evidence of this mode has been observed by Belle:

$$
\left.R_{A D S} \equiv \frac{N\left(B^{+} \rightarrow\left(K^{-} \pi^{+}\right)_{D} K^{+}\right)+C . C .}{N\left(B^{+} \rightarrow\left(K^{+} \pi^{-}\right)_{D} K^{+}\right)+C . C .}=1.62 \pm 0.42_{-0.19}^{+0.16} \quad \text { (Belle }\right) .
$$

When all relevant $D K$ modes are combined, the ADS method is expected to lead to $\sigma_{\phi_{3} / \gamma}$ of less than $10^{\circ}$ using the data currently available. 